as a bulking agent around the urethra, the Macroplastique Implantation System (MIS; Uroplasty BV, Geleen, The Netherlands) provides an alternative to standard endoscopic silicon implantation.

This prospective evaluation included 21 consecutive women with urodynamically diagnosed intrinsic sphincter deficiency. All were treated using the MIS system and there were eight re-treatments within the first 3 months of follow-up. The procedure was performed under local anesthesia on an outpatient basis, using a mean silicone elastomer volume of $6.3 \mathrm{ml}$.

Two years after the last injection, 10 patients considered themselves cured and three felt that their condition had improved. The surgeons' subjective assessments were similar, with eight patients considered to be cured and five to have improved. Eight cases $(38.1 \%)$ were considered a failure by both the patients and the surgeon. Objective assessment using the pad weight test showed a failure rate of $33.3 \%$, whereas urodynamic testing indicated that only five (25\%) patients' treatments had failed. Quality-of-life improved significantly in all domains of the King's Health Questionnaire.

The authors conclude that the MIS procedure was safe and easy to perform, and was successful in more than $60 \%$ of cases. They note that strict patient selection was an important factor in achieving these results.

Original article Tamanini JTN et al. (2004) Treatment of intrinsic sphincter deficiency using the macroplastic implantation system: two-year follow-up. J Endourol 18: 906-911

\section{Advances in thermal therapy for recurrent prostate cancer}

Interstitial microwave thermal therapy has been investigated as a treatment for certain solid tumors. Sherar et al. have applied this technology to the field of prostate cancer and have recently reported on their experience.

Thermal therapy is based on the selective coagulation of the tumor tissue; protection of the surrounding, normal tissue is crucial. Interstitial microwave therapy allows relatively precise control of the zone of thermal damage. Indeed, treatment simulations using phantom materials showed that the prostate can be targeted whilst the urethra is protected using a cooling mechanism. A Phase I/II trial examined the safety and efficacy of interstitial microwave therapy as an alternative to prostatectomy or hormone therapy in 41 patients with prostate cancer. All patients had recurrent disease following external beam radiation therapy. Disease-free survival was similar to that found using cryotherapy but, importantly, complication rates were lower.

The authors explain that the development of this technology will focus on the prediction of changes in tissue properties and blood flow during the heating process. It will also be important to determine the optimal level of protection of the normal tissue and to reduce cost and risk. Noting that the treatment might be appropriate for other patient groups, including those who have failed primary brachytherapy, Sherar et al. call for larger, multicenter trials in this area.

Original article Sherar MD et al. (2004) Interstitial microwave thermal therapy and its application to the treatment of recurrent prostate cancer. Int J Hyperthermia 20: 757-768 